\title{
Spatiotemporal regulation of endogenous MSCs using a functional injectable hydrogel system for cartilage regeneration
}

\author{
Yunsheng Dong ${ }^{1}$, Yufei Liu', Yuehua Chen ${ }^{1}$, Xun Sun ${ }^{2}$, Lin Zhang ${ }^{1}$, Zhiling Zhang ${ }^{3}$, Yanying Wang ${ }^{3}$, Chunxiao Qi', \\ Shufang Wang (1D) and Qiang Yang (1) ${ }^{2}$
}

\begin{abstract}
Hydrogels have been extensively favored as drug and cell carriers for the repair of knee cartilage defects. Recruiting mesenchymal stem cells (MSCs) in situ to the defect region could reduce the risk of contamination during cell delivery, which is a highly promising strategy to enhance cartilage repair. Here, a cell-free cartilage tissue engineering (TE) system was developed by applying an injectable chitosan/silk fibroin hydrogel. The hydrogel system could release first stromal cell-derived factor-1 (SDF-1) and then kartogenin (KGN) in a unique sequential drug release mode, which could spatiotemporally promote the recruitment and chondrogenic differentiation of MSCs. This system showed good performance when formulated with SDF-1 $(200 \mathrm{ng} / \mathrm{mL})$ and PLGA microspheres loaded with KGN $(10 \mu \mathrm{M})$. The results showed that the hydrogel had good injectability and a reticular porous structure. The microspheres were distributed uniformly in the hydrogel and permitted the sequential release of SDF-1 and KGN. The results of in vitro experiments showed that the hydrogel system had good cytocompatibility and promoted the migration and differentiation of MSCs into chondrocytes. In vivo experiments on articular cartilage defects in rabbits showed that the cell-free hydrogel system was beneficial for cartilage regeneration. Therefore, the composite hydrogel system shows potential for application in cell-free cartilage TE.
\end{abstract}

\section{Introduction}

Articular cartilage (AC) plays important roles in load bearing, mechanical stress resistance and shock absorption $^{1,2}$. Once cartilage has been damaged, selfhealing is difficult due to a lack of vascular tissue for adequate nutritional supply ${ }^{3,4}$. Trauma, disease, and cartilage degeneration often lead to cartilage defects ${ }^{5}$. If cartilage defects are not treated adequately, the joints will show irreversible deterioration, even leading to disability. Current treatment methods, such as

\footnotetext{
Correspondence: Shufang Wang (wangshufang@nankai.edu.cn) or Qiang Yang (yangqiang1980@126.com)

${ }^{1}$ State Key Laboratory of Medicinal Chemical Biology; The Key Laboratory of Bioactive Materials, The College of Life Science, Nankai University, 300071 Tianjin, China

${ }^{2}$ Department of Spine Surgery, Tianjin Hospital, Tianjin University, 300211 Tianjin, China

Full list of author information is available at the end of the article
}

microfracture, autogenous chondrocyte transplantation, and allogeneic osteochondral transplantation, can reduce pain and improve joint function ${ }^{6-8}$. However, they lack stable integration with adjacent host tissues and result in fibrocartilage formation, adversely affecting the quality and function of regenerated cartilage, thereby leading to further complications ${ }^{9}$. Tissue engineering (TE) is an up-and-coming strategy to achieve effective cartilage regeneration.

A significant target of TE is the construction of biomimetic extracellular matrix (ECM) biomaterials, which can provide mechanical support and a suitable microenvironment for cell survival. Hydrogels are very good ECM biomimetic materials with three-dimensional crosslinked structures and high water contents ${ }^{10}$. In particular, injectable hydrogels used as drug carriers can be easily adapted to defects of any size or shape and allow the

\section{(c) The Author(s) 2021}

(c) Open Access This article is licensed under a Creative Commons Attribution 4.0 International License, which permits use, sharing, adaptation, distribution and reproduction cc) in any medium or format, as long as you give appropriate credit to the original author(s) and the source, provide a link to the Creative Commons license, and indicate if changes were made. The images or other third party material in this article are included in the article's Creative Commons license, unless indicated otherwise in a credit line to the material. If material is not included in the article's Creative Commons license and your intended use is not permitted by statutory regulation or exceeds the permitted use, you will need to obtain permission directly from the copyright holder. To view a copy of this license, visit http://creativecommons.org/licenses/by/4.0/. 
even distribution of drugs ${ }^{11-13}$. Natural materials and synthetic materials have unique advantages in the preparation of hydrogels ${ }^{14}$. Chitosan (CS), a carbohydrate biopolymer, has an inherent linear structure, good biocompatibility, and good biodegradability, and it allows easy chemical modification ${ }^{15,16}$. It has a structure similar to that of glycosaminoglycans (GAGs, a component of ECM), which is conducive to cell adhesion ${ }^{17}$. However, CS has poor mechanical properties that cannot meet the needs of clinical application, so it is necessary to combine CS with other materials to compensate for its shortcomings $^{18}$. Silk fibroin (SF), a natural material with good mechanical properties ${ }^{19}$, and chitosan are expected to form biomaterials with good mechanical properties.

In addition to biomaterials, bioactive molecules are important factors in TE and have unique advantages in promoting the chondrogenic differentiation of bone marrow mesenchymal stem cells (BMSCs) and enhancing cartilage regeneration ${ }^{20,21}$. The combination of multiple drugs for sequential release represents a novel strategy for cartilage $\mathrm{TE}^{22,23}$. Stromal cell-derived factor-1 (SDF-1) can be recognized by the CXCR4 receptor to promote the migration of CXCR4-positive progenitors and stem cells to injury sites ${ }^{24,25}$. Kartogenin (KGN) is a small-molecule compound first discovered by Johnson et al. in $2012^{26,27}$ that can induce mesenchymal stem cells (MSCs) to differentiate into cartilage and protect cartilage by regulating the CBF- $\beta /$ Runx1 signaling pathway ${ }^{28,29}$. Therefore, it has obvious advantages in cartilage TE. However, the lymphatic system rapidly clears KGN injected via the articular cavity. In addition, intra-articular injection of KGN can lead to organ damage due to leakage into the circulatory sys$t^{3} \mathrm{~m}^{30,31}$. Therefore, a delivery system for the controlled release of KGN is urgently required. Microspheres have been widely used in cartilage TE for drug delivery ${ }^{32}$. Sun et al. prepared collagen scaffolds loaded with KGN microspheres, which can release KGN in a controlled manner to promote cartilage regeneration ${ }^{33}$.

Chondrocytes, the most common cell source for transplantation, could lead to immune rejection and dedifferentiation $^{34}$. MSCs are under consideration as another cell source for tissue regeneration ${ }^{35}$. In particular, endogenous MSCs have attracted much attention in cartilage repair ${ }^{36}$. In addition, bone marrow has been proven to be a potential MSC source reservoir that contributes to the regenerative capacity of other tissues ${ }^{37,38}$. However, endogenous MSCs could bring about a poor repair effect because of their poor directed migration ${ }^{39}$ and the failure of chondrogenic differentiation ${ }^{40}$. Therefore, it is worth targeting bone-marrowderived MSCs to effectively home to the site of injury and enhance their chondrogenic effect.

In this study, we developed a p-hydroxybenzene propanoic acid (PA)-modified chitosan (PC)/SF hydrogel containing SDF-1 and microspheres loaded with KGN.
The hydrogel facilitated sequential drug release. SDF-1 was initially released from the hydrogel to facilitate the recruitment of rBMSCs in vivo. Subsequently, KGN was released slowly from microspheres in the hydrogel and promoted the chondrogenesis of MSCs. The injectable hydrogel was suitable for irregular or deep cartilage defects and was closer to the edge of the defect. With the combination of physically mixed SDF- 1 and microspheres encapsulated with KGN in the hydrogel, we expected enhanced synergistic effects for cartilage regeneration, in which the rapid initial release of SDF-1 facilitates the recruitment of MSCs, and the controlled release of KGN subsequently reduces side effects and induces the chondrogenic differentiation of MSCs. The effects of the sequential release of SDF-1 and KGN on the migratory and chondrogenic capacity of MSCs and cartilage regeneration were assessed. The research strategy is shown in Fig. 1. We plan to establish a cell-free TE hydrogel to achieve the sequential release of multiple drugs to enhance cartilage regeneration, which could provide a new strategy for articular cartilage regeneration.

\section{Experimental section}

\section{The extraction of SF solution}

SF solution was extracted as previously described ${ }^{4}$. In brief, SF fiber was obtained from Bombyx mori silkworm silk by removing sericin with sodium carbonate, and then SF solution was obtained by dissolving SF fiber with lithium bromide, subjected to dialysis with distilled water and concentrated with polyethylene glycol.

\section{Synthesis of $p$-hydroxybenzene propanoic acid-modified chitosan}

PC was synthesized by adding $0.5 \mathrm{~g}$ of CS and $0.205 \mathrm{~g}$ of PA to a $100 \mathrm{~mL}$ beaker. Then, $50 \mathrm{~mL}$ of distilled water and $0.25 \mathrm{~mL}$ of acetic acid were added to the beaker and stirred for $6 \mathrm{~h}$ at room temperature. Subsequently, $0.173 \mathrm{~g}$ of N-hydroxy succinimide (NHS) and $0.363 \mathrm{mg}$ of 1-ethyl-3-(3dimethylaminopropyl)carbodiimide hydrochloride (EDC) were added to the reaction solution, and $48 \mathrm{~h}$ was allowed to complete the reaction. The product was obtained by lyophilization procedures after dialysis for 3 days.

\section{Characterization of $p$-hydroxybenzene propanoic acid- modified chitosan}

${ }^{1} \mathrm{H}$-NMR spectra of the samples dissolved in deuterium oxide were collected by a Bruker AV400 NMR spectrometer using PA as a standard. FTIR spectra were obtained from 4000 to $400 \mathrm{~cm}^{-1}$ on a Bio-Rad FTS6000 Fourier transform spectrometer.

\section{Preparation of KGN-loaded microspheres}

One milliliter of PLGA solution $(0.2 \mathrm{~g} / \mathrm{mL}$ in dichloromethane, lactide:glycolide $\left.=50: 50, \quad M_{\mathrm{w}}=20 \mathrm{kDa}\right)$ and 


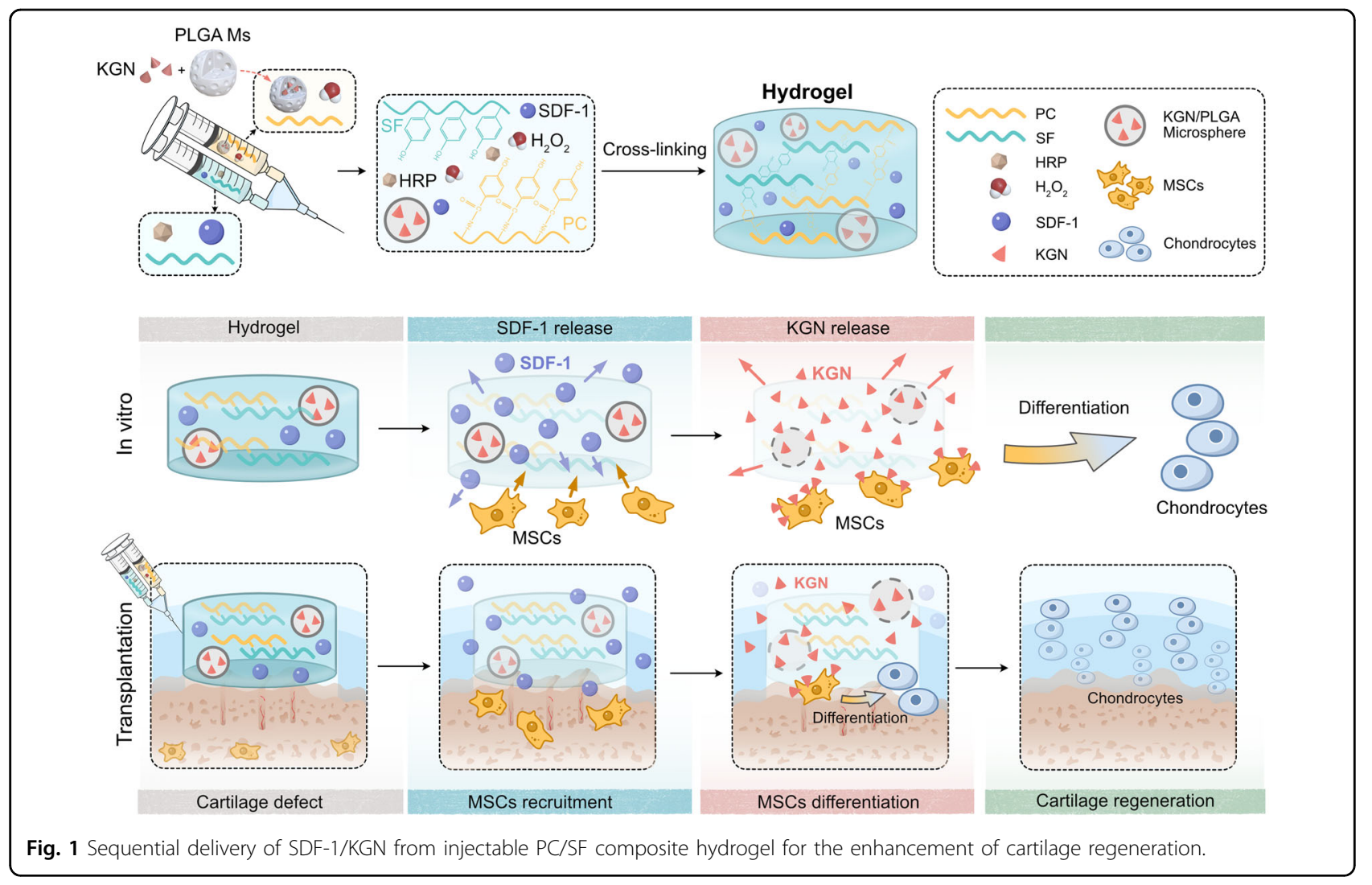

$0.1 \mathrm{~mL}$ of $\mathrm{KGN}$ solution $(50 \mathrm{mg} / \mathrm{mL}$ in dimethyl sulfoxide) were mixed to obtain the oil phase. After full dissolution, the oil phase was dropped into $30 \mathrm{~mL}$ of polyvinyl alcohol solution (PVA, $0.02 \mathrm{~g} / \mathrm{mL}$ ), and the mixture was emulsified at 15,000 rpm for $3 \mathrm{~min}$. After solvent evaporation for $6 \mathrm{~h}$, the pellets were washed three times, and KGN-loaded PLGA microspheres were obtained after freeze-drying.

\section{Characters of KGN-loaded PLGA microspheres Microsphere morphology}

A scanning electron microscope (SEM, QUANTA 200) obtained from Thermo Fisher Scientific in USA was used to observe the microstructure of the microspheres. ImageJ software was used to calculate the average value of microspheres $(n=30)$ as their mean particle size.

\section{Loading and encapsulation rates of microspheres}

The content of KGN (M1) in PLGA microspheres (M0) was determined by high-performance liquid chromatography (HPLC, Agilent 1260 LC, $n=3$ ) after the microspheres were destroyed by sodium hydroxide solution. The loading rate (LR, \%) was calculated according to the equation "M1/M0 $\times 100 \%$ ", and the encapsulation rate (ER, \%) of KGN was obtained following the equation "LR/ theoretical loading efficiency $\times 100 \% "$.

\section{KGN release behavior of microspheres}

The release characteristics of PLGA microspheres (per unit volume of microsphere solution containing 1,10 , and $100 \mu \mathrm{M} \mathrm{KGN}, n=3)$ were obtained by measuring the KGN content in the release medium $(1.1 \mathrm{~mL})$ at specific times by HPLC.

\section{Fabrication of injectable PC/SF hydrogel with sequential release of SDF-1 and KGN}

After the preparation and characterization of different ratios of silk fibroin (SF) and PC for hydrogels by the method in Supplementary Experimental Section 1.2, the optimum ratio of injectable $\mathrm{PC} / \mathrm{SF}$ hydrogel with sequential release SDF-1 and KGN was determined. Briefly, a $5 \%$ SF solution containing horseradish peroxidase (HRP) (300 units, $10 \mathrm{mg} / \mathrm{mL}$ ) with/without SDF-1 ( $400 \mathrm{ng} / \mathrm{mL}$ PA solution) was prepared as solution A. Then, a 1\% PC solution containing hydrogen peroxide with/without KGN-loaded PLGA microspheres (0, 1, 10, $100 \mu \mathrm{M}$ KGN) was prepared as solution B. Solution A and solution $\mathrm{B}$ were mixed equally by a double syringe to form hydrogels with/without SDF-1 and KGN. The experiment was divided into five groups: PSH (hydrogel without SDF1 and microspheres), SPSH (hydrogel containing SDF-1 without microspheres), KPSH10 (hydrogel without SDF-1 containing $100 \mu \mathrm{M}$ KGN-loaded microspheres), SKPSH1 
(hydrogel containing SDF-1 and $1 \mu \mathrm{M}$ KGN-loaded microspheres), SKPSH10 (hydrogel containing SDF-1 and $10 \mu \mathrm{M}$ KGN-loaded microspheres), and SKPSH100 (hydrogel containing SDF-1 and $100 \mu \mathrm{M}$ KGN-loaded microspheres).

\section{Characterization of injectable PC/SF hydrogel with sequential release of SDF-1 and KGN Hydrogel morphological characteristics}

The micromorphology was observed by SEM, and the pore size was calculated by ImageJ software.

\section{Hydrogel rheological properties}

A rotational rheometer was used to measure the rheological properties of the hydrogels. The diameter of the rotor was $20 \mathrm{~mm}$. The test temperature was $37^{\circ} \mathrm{C}$, and the fixed angle frequency was $1 \mathrm{rad} / \mathrm{s}$. After $20 \mathrm{~min}$ of detection, the elastic modulus ( $\left.G^{\prime}\right)$ and the viscous modulus $(G ")$ of the hydrogel were obtained.

\section{SDF-1 and KGN release profiles of hydrogels}

The release characteristics of SDF-1 and KGN in the hydrogels $(1 \mathrm{~mL}, n=3)$ were obtained by measuring their content in the release medium $(1.1 \mathrm{~mL})$ at specific times by ELISA and HPLC, respectively.

\section{Evaluation of cellular vitality in hydrogels}

After 1 and 7 days in culture, the viability of rBMSCs (Cyagen, China) at a density of $1 \times 10^{6}$ cells $/ \mathrm{mL}$ seeded into hydrogel was evaluated by staining the cells with the Live/Dead kit. Next, laser confocal microscopy (LSM710; Zeiss, Oberkochen, Germany) was performed to observe dead cells (red) and living cells (green). The quantitative analysis of cell viability was performed by cell counting kit- 8 (CCK- 8$)$ at 1 and 7 days. The experiment was divided into five groups (control: hydrogel without cells, PSH: hydrogel+cells, SPSH: hydrogel with SDF-1+cells, KPSH10: hydrogel with $10 \mu \mathrm{M}$ KGN-loaded microspheres + cells, SKPSH10: hydrogel with SDF-1 and $10 \mu \mathrm{M}$ KGNloaded microspheres+cells).

After 1 day in culture, the rBMSCs seeded into hydrogel at a density of $1 \times 10^{6}$ cells $/ \mathrm{mL}$ were fixed and dehydrated. Next, SEM was performed to observe the adhesion of cells on the hydrogel.

\section{Bioactivity assessment of SDF-1 and KGN from hydrogels in vitro}

\section{Migration of $r B M S C s$ in vitro}

Aliquots of $200 \mu \mathrm{L}$ of rBMSCs $\left(5 \times 10^{5}\right.$ cells $\left./ \mathrm{mL}\right)$ were seeded into the upper chambers with $8 \mu \mathrm{m}$ pore size of 24-well Transwell plates. Then, $0.6 \mathrm{~mL}$ of $\alpha$-MEM medium (containing 2\% fetal bovine serum (FBS) and 1\% penicillin-streptomycin) was added to the lower chamber and cultured for $12 \mathrm{~h}$. Subsequently, a thin layer of hydrogel was coated on the bottom of the lower chamber, the medium was replaced with serum-free medium, and incubation was continued for an additional $12 \mathrm{~h}$. After being fixed, the cells were stained with $0.1 \%$ crystal violet for $15 \mathrm{~min}$ and washed with PBS three times for $5 \mathrm{~min}$ each time to remove residual crystal violet. The migrated cells were photographed under a microscope and quantified using ImageJ (National Institutes of Health). The TCP lower chamber without hydrogel served as a control group.

\section{Induction of chondrogenic differentiation of rBMSCs}

The rBMSCs $\left(1 \times 10^{6}\right.$ cells $/ \mathrm{mL}$, passage $\left.=3\right)$ in induction medium (without chondrogenic growth factors) were cultured in hydrogels for 7 or 21 days to evaluate chondrogenic induction. The induction medium was changed every 3 days. The chondrogenic induction of rBMSCs in hydrogel was confirmed by histological staining to determine the distribution of cells (hematoxylin and eosin, $\mathrm{H} \& \mathrm{E}$ ) and the production of GAGs (toluidine blue/ safranin-O) and by immunohistochemical staining to determine the production of collagen II (COL-II, 1:100, NB600-844, Novus). The quantitative analysis of GAGs and COL-II in the hydrogels was performed by an enzyme-linked immunosorbent assay kit (Wuhan ColorfulGene Biological Technology, Wuhan, China) after induction culture for 7 or 21 days.

The effects of chondrogenic differentiation of rBMSCs were further evaluated at the gene expression level by qRT-PCR using primers (Table S1) for the transcription factors SOX-9, aggrecan (ACAN) and COL-II. In brief, after 21 days of cultivation in hydrogels, the total RNA of samples in each group was extracted and quantified using a Nucleic Acid and Protein Analyzer (Nanodrop 2000, Thermo Fisher Scientific). One microgram of total RNA was used for cDNA synthesis by the PrimeScript ${ }^{\mathrm{TM}}$ RT Reagent Kit with gDNA Eraser (Takara, RR047A). The primer sequences, qPCR SYBR ${ }^{\circledR}$ Green Master Mix (Yeasen) and cDNA $(0.4 \mu \mathrm{g})$ were used for quantitative RT-PCR analysis. The relative gene expression was normalized to glyceraldehyde 3-phosphate dehydrogenase (GAPDH) expression and presented as the fold-change compared to the PSH group using the $\Delta \Delta \mathrm{Ct}$ method.

\section{Cartilage regeneration in vivo Animal model}

The animal model was established with the approval of the Animal Experimental Ethical Inspection Committee of Nankai University. Briefly, New Zealand white rabbits (2.2-2.5 kg, 3 rabbits, $n=6$ knees per group for each time point) were anesthetized with xylazine $(20 \mathrm{mg} / \mathrm{kg}$ body weight) and midazolam ( $0.8 \mathrm{mg} / \mathrm{kg}$ body weight) by intramuscular injection. A cartilage defect (diameter $=$ $4 \mathrm{~mm}$, depth $=1.5 \mathrm{~mm}$ ) combined with microfracture 

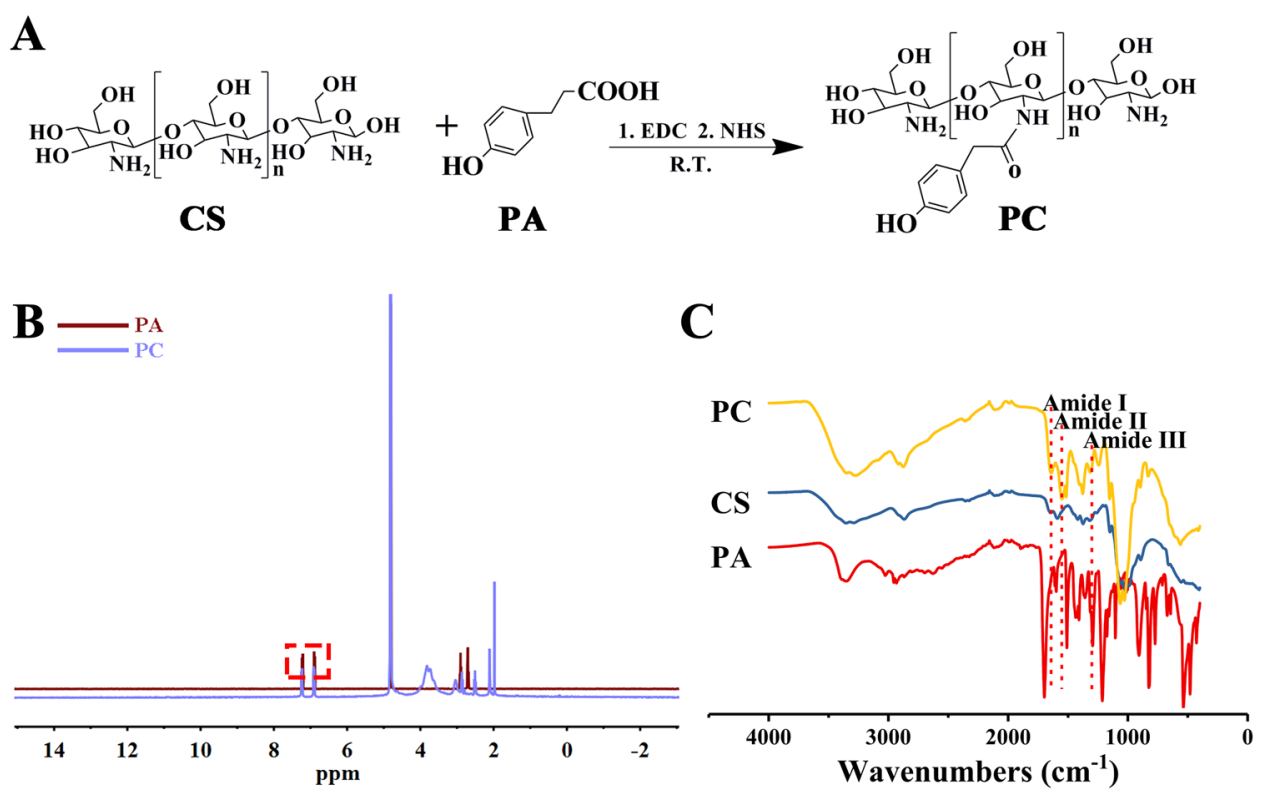

Fig. 2 Reaction route and characterization of PC. A Reaction route, ${ }^{1}{ }^{1}$-NMR, C FTIR.

(MF) was made in the trochlear groove of both knee joints of animals (Fig. S1A). Then, the hydrogels (PSH, SPSH, KPSH10 and SKPSH10) were injected into the defect sites, as shown in Fig. S1B. MF alone was used as a control group. After treatments, the knee joint capsule and the skin were sutured in layers. After the operation, all rabbits were allowed to move freely, penicillin (80000 U) was administered up to 3 days, 4 weeks, and 12 weeks after implantation, and the knees were harvested for further evaluation.

\section{Evaluation of cartilage repair}

At 1 week postoperatively, samples were assessed by immunofluorescence staining for CD44 (1:100, NBP222530F, Novus) and CD90 (1:100, ab225, Abcam) to identify endogenous MSC recruitment. Detailed experimental procedures can be found in the supporting information.

At 4 and 12 weeks postoperatively, samples were assessed by macroscopic observation according to the criteria of the International Cartilage Repair Society (ICRS, Table S2), by immunofluorescence staining for CD44 (1:100, NBP2-22530F, Novus) and CD90 (1:100, ab225, Abcam) to identify endogenous MSC recruitment, $H \& E$ staining for the morphology and cell distribution of regenerated cartilage, and safranin-O/fast green staining for ACAN and by immunohistochemical analysis for COL-II (1:100, NB600-844, Novus) deposition. Detailed experimental procedures can be found in the supporting information. To evaluate the progress of cartilage repair, sections at 4 and 12 weeks were blindly scored by three independent observers according to an established scoring system (Table S3).

\section{Statistical analysis}

Data are presented as the mean \pm standard deviation (SD). Comparisons between groups were performed using Student's $t$-test and Tukey's test. In all analyses, $p<0.05$ was taken to indicate statistical significance. All experiments were repeated at least three times.

\section{Results}

Characterization of $p$-hydroxybenzene propanoic acidmodified chitosan

The route of PC synthesis is shown in Fig. 2A. The carboxyl groups in the PA structure were activated using EDC and NHS and reacted with the amino groups in CS to form amide bonds, thus producing PC. The PC was then subjected to ${ }^{1} \mathrm{H}-\mathrm{NMR}$ spectroscopy (Fig. 2B) and infrared spectroscopy (Fig. 2C). ${ }^{1} \mathrm{H}-\mathrm{NMR}$ showed that the chemical shifts of $\delta 7.11-7.13$ and $\delta 6.79-6.81$ in PA spectra were attributable to proton absorption peaks at intersite and ortho sites of the benzene ring, indicating that PA was successfully grafted into the CS molecular chain. The absorption peaks in the infrared spectrum of PC at 1643, 1550, and $1243 \mathrm{~cm}^{-1}$ belonged to amide I, amide II, and amide III, further indicating that the carboxyl groups of PA reacted with the amino groups of CS, and PA was grafted to the molecular chain of CS to form PC (Fig. 2C).

PLGA microspheres with sustainable release of KGN were prepared by the single emulsion method. The PLGA microspheres had an obviously spherical morphology and 

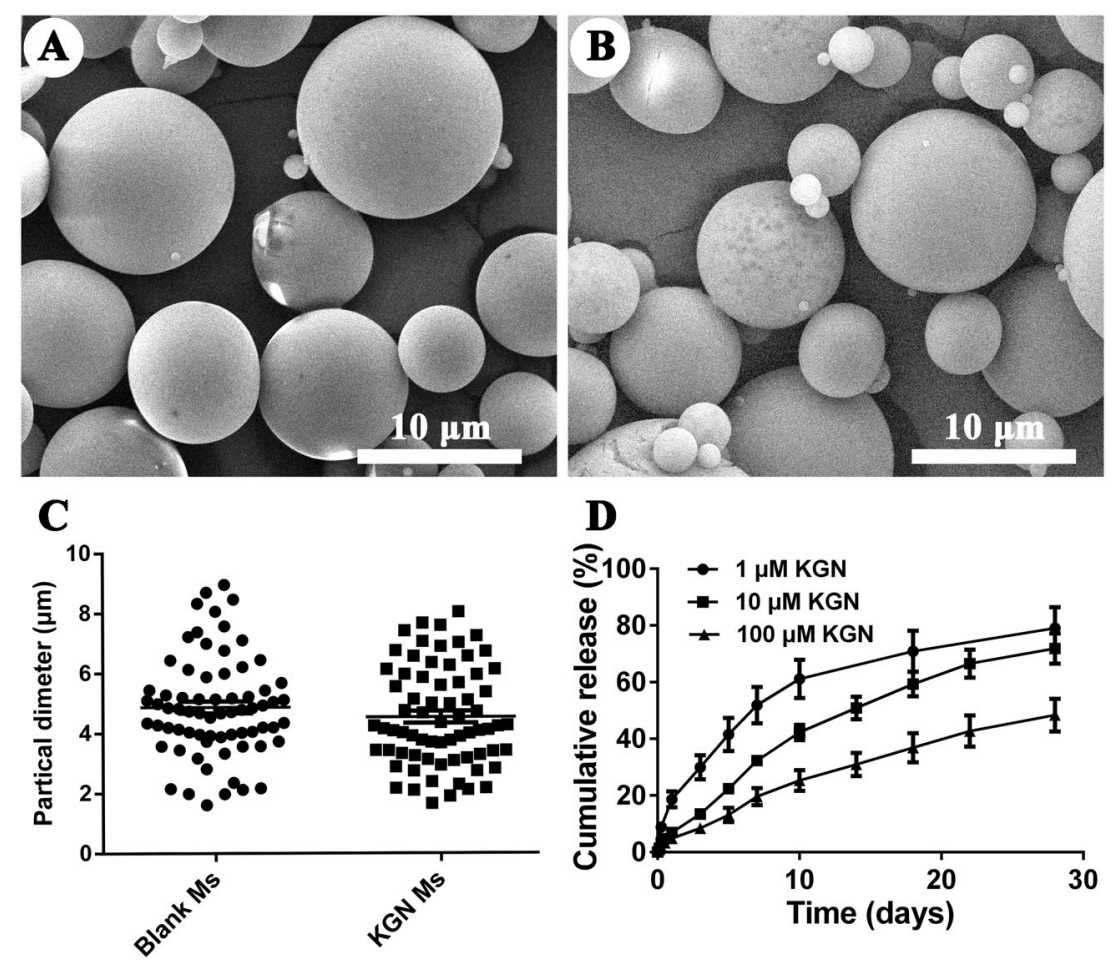

Fig. 3 Characterization of microspheres. Micromorphology of A blank microspheres and $\mathbf{B}$ microspheres loaded with KGN. C Particle size analysis of microspheres. D Cumulative release of KGN from microspheres.

Table 1 Drug loading and encapsulation rate of PLGA microspheres for KGN by HPLC.

\begin{tabular}{lll}
\hline Sample & $\begin{array}{l}\text { Loading efficiency (mg KGN/mg } \\
\text { PLGA microspheres) }\end{array}$ & $\begin{array}{l}\text { Encapsulation } \\
\text { efficiency (\%) }\end{array}$ \\
\hline KGN Ms & $0.0175 \pm 0.0009$ & $69.87 \pm 3.7 \%$ \\
\hline
\end{tabular}

smooth surface (Fig. 3A and B). The particle sizes of unloaded and loaded microspheres were $4.87 \pm 1.68 \mu \mathrm{m}$ and $4.53 \pm 1.65 \mu \mathrm{m}$, respectively (Fig. $3 \mathrm{C}$ ). The results showed that the loading of KGN had no significant effects on the diameter of the prepared microspheres. The loading efficiency and encapsulation efficiency of KGN in PLGA microspheres, as shown in Table 1, were $0.0175 \pm$ 0.0009 and $69.87 \pm 3.7 \%$, respectively. The results shown in Fig. 3D indicated that the KGN release rates from microspheres loaded with KGN at concentrations of 1,10 , and $100 \mu \mathrm{M}$ were $18.65 \pm 2.78 \%, 6.99 \pm 2.39 \%$, and $4.86 \pm$ $1.66 \%$ at $24 \mathrm{~h}$, respectively, indicating marked burst release from microspheres loaded with $1 \mu \mathrm{M} \mathrm{KGN}$, while those loaded with $10 \mu \mathrm{M}$ and $100 \mu \mathrm{M}$ KGN showed no obvious burst release within $24 \mathrm{~h}$. The release rates of the microspheres with the three different KGN concentrations were $79.01 \pm 7.44 \%, 71.89 \pm 5.35 \%$, and $48.35 \pm$
$5.78 \%$, respectively, after 28 days. Therefore, KGN could be released slowly by microspheres.

\section{Characterizations of injectable hydrogels}

The 1:5 mass ratio of $\mathrm{PC} / \mathrm{SF}$ for hydrogels with sequential release of SDF-1 and KGN was determined by the preparation and characterization (Fig. S2-S6) of hydrogels with different ratios (mass ratios of 1:0, 5:1, 1:1, $1: 5$, and $1: 10$ for $\mathrm{PC}$ and $\mathrm{SF}$ ). The results indicated that the PC/SF (mass ratios of 1:0, 5:1, 1:1, and 1:5) hydrogel had good gelling performance (Fig. S2), injectability (Fig. S3) and porous structures $\mathrm{s}$ (Fig. S4), and the PC/SF solutions with a mass ratio of $1: 5$ also had good stability and mechanical properties (Figs. S5-S6). The PC/SF mixed solutions with/without SDF-1 and microspheres were extruded into different shapes to evaluate their injectability. The results presented in Fig. 4A1-D1 show that the mixed solutions in all groups could form hydrogels with good injectability, which were not affected by the addition of microspheres and drugs. Microstructural observation (Fig. 4A2-D2, Fig. 4A3-D3, and Fig. S7) showed that the hydrogels had porous network structures, and the microspheres were evenly distributed in the hydrogel. The porous structures did not change significantly with increases in the sizes of microspheres and were similar to the structure of the ECM, which is beneficial 


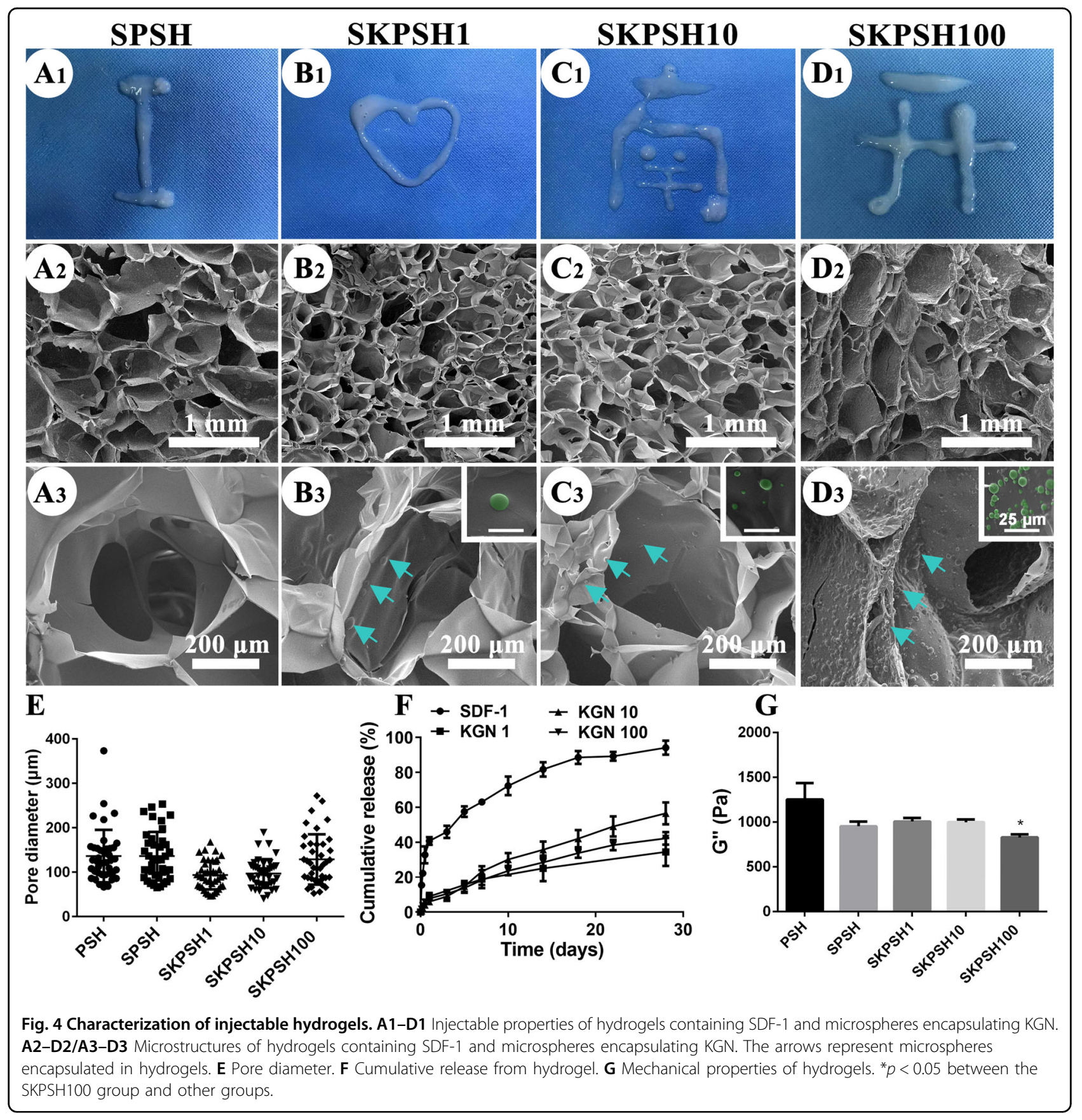

for cell adhesion, proliferation and differentiation. The PSH, SPSH, SKPSH1, SKPSH10, and SKPSH100 groups had pore sizes of $135.74 \pm 55.89,136.04 \pm 54.38,93.48 \pm 32.18$, $96.91 \pm 32.32$, and $128.94 \pm 56.7 \mu \mathrm{m}$, respectively (Fig. 4E). The drug and microspheres had no effects on the hydrogel pore sizes.

The release profiles of SDF-1 and KGN from the PC/SF injectable hydrogel in vitro were investigated, and the results are presented in Fig. 4F. SDF-1 showed an obvious burst and rapid release, with release rates of $40.68 \pm 2.19 \%$ at $24 \mathrm{~h}$; the release subsequently slowed, with $\sim 60 \%$ of the total SDF-1 released after $7 \mathrm{~d}$. In contrast, the overall release of KGN from the hydrogel was comparatively slow and sustained compared with that of SDF-1; the microspheres loaded with 1,10 , and $100 \mu \mathrm{M}$ KGN showed sustained release rates of $8.99 \pm 2.02 \%, 5.97 \pm 1.85 \%$, and $7.79 \pm 1.57 \%$, respectively, at $24 \mathrm{~h}$ (Fig. $4 \mathrm{~F}$ and Fig. S8A) and of $34.45 \pm 7.99 \%, 56.53 \pm 6.29 \%$, and $42.17 \pm 3.62 \%$, respectively, at 28 days (Fig. $4 \mathrm{~F}$ and Fig. S8B) in all groups.

The KGN was released slowly for as long as 4 weeks. SDF-1 was released rapidly at the initial stage and throughout the release process, while KGN was released 
slowly. Therefore, SDF-1 and KGN have obvious sequential release behavior, indicating the spatiotemporally different release behavior of the two bioactive molecules from the hydrogels.

SDF-1 and KGN were loaded into hydrogels by physical blending and microsphere encapsulation strategies, resulting in sequential release behaviors. Moreover, the SKPSH100 group showed obviously reduced mechanical properties with the excessive addition of microspheres (Fig. 4G and Fig. S9). SKPSH100 was not suitable for subsequent experiments. Furthermore, the renewal of body fluids may reduce the concentration of the released drug, so we should select a higher concentration for evaluation in vivo to ensure that it retains good biological function. Therefore, the hydrogel containing $10 \mu \mathrm{M}$ KGN was more suitable for practical applications.

\section{Evaluation of cellular compatibility of hydrogels}

The biocompatibility of hydrogel formulations was studied by evaluating the adhesion and viability of rBMSCs. The results of SEM observations indicated that the hydrogel formulations were conducive to rBMSC adhesion (Fig. S10). The viability of cells seeded onto hydrogel was evaluated by live/dead cell staining (Fig. 5A and Fig. S11) and CCK-8 (Fig. 5B and C). The live/dead cell staining results showed that the cells in the hydrogel were stained green, which indicated that hydrogels are good for cell survival. The quantitative results further revealed that the cells in the hydrogel had high activity, and there was no significant difference among the groups, but the SPSH group and SKPSH group showed a trend of increasing activity at 7 days. This may be related to the function of SDF-1 in promoting cell proliferation. These results indicated that the hydrogel had good cytocompatibility.

\section{The rBMSCs migration in vitro}

A Transwell system was used to evaluate the activity of SDF-1 released from hydrogel to recruit rBMSCs in vitro. The transmembrane migration of cells was determined in the control, PSH, SPSH, KPSH10, and SKPSH10 groups (Fig. 6). The numbers of migrating rBMSCs in the SDF-1 groups (SPSH and SKPSH10 groups) were greater than those in the control, PSH and KPSH10 groups; although the average number of migrating cells of SKPSH10 was slightly greater than that of SPSH, there was no significant difference between the SPSH and SKPSH10 groups. This may be related to the function of KGN in promoting cell proliferation to some extent ${ }^{41}$. Thus, the results showed that the SDF-1 released from the hydrogel had favorable bioactivity and could promote rBMSC recruitment without the influence of KGN.

\section{Chondrogenesis of rBMSCs in vitro}

The chondrogenic differentiation of rBMSCs in hydrogels was evaluated at different incubation times (Fig. 7). H\&E staining (Fig. 7A) showed that the hydrogel was beneficial for cell infiltration and proliferation. Positive
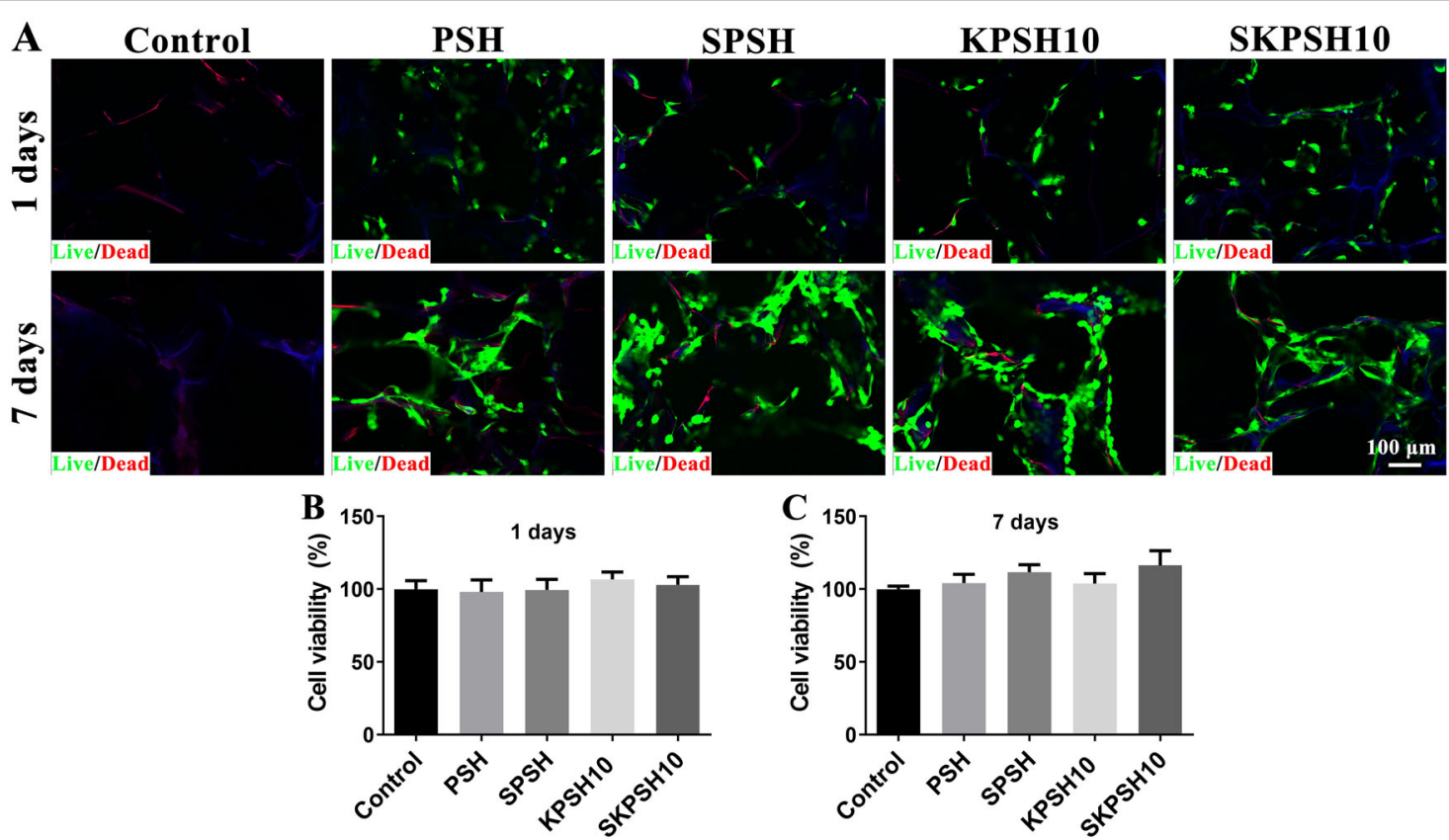

Fig. 5 Cellular compatibility assessment for hydrogel by live/dead staining and CCK-8. Evaluation of rBMSC viability in hydrogels by $\mathbf{A}$ live/ dead cell staining and B, C CCK-8 assay at 1 and 7 days. 
Transwell insert

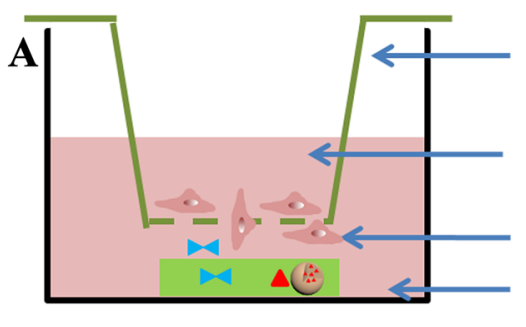

Upper compartment

Migrated cells

Lower compartment

\section{$\triangle$ KGN Hydrogel}

BMSCs \ SDF-1

Microsphere
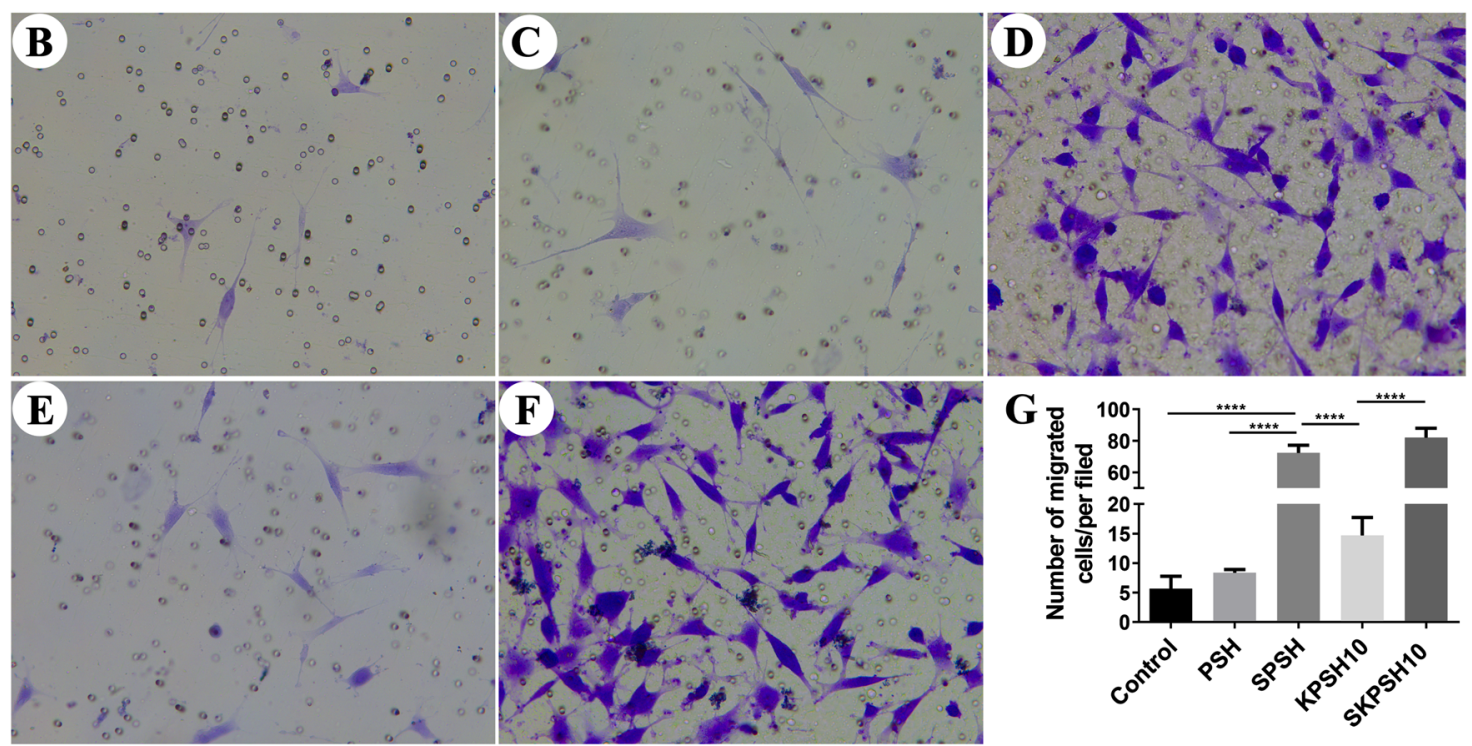

Fig. 6 Migration of rBMSCs in vitro. A Schematic diagram. B Control. C PSH. D SPSH. E KPSH10. F SKPSH10. G Quantitative analysis of migrated cells.

staining was more obvious in the KPSH10 and SKPSH10 groups, indicating that the ECM content was significantly higher in these two groups than in the control, PSH and SPSH groups after 21 days. With increasing culture time, KPSH10 and SKPSH10 showed increased positive staining with toluidine blue and safranin-O as well as immunohistological staining (Fig. 7A) for COL-II, indicating that hydrogels containing KGN promoted ECM formation. Furthermore, the results of quantitative analysis (Fig. 7B and $\mathrm{C}$ ) indicated that the hydrogels containing KGN (KPSH10 and SKPSH10 groups) could enhance GAG and COL-II formation. Thus, the KGN released from hydrogel in vitro had effective bioactivity and could promote the chondrogenic differentiation of rBMSCs. The qRT-PCR results indicated the upregulation of chondrogenic genes at 21 days due to the release of KGN in the KPSH10 and SKPSH10 groups compared to the PSH and SPSH groups (Fig. 8).Specifically, the COLII, ACAN, and SOX-9 mRNA levels were $27.66 \pm 4.19$, $11.99 \pm 0.94$, and $12.85 \pm 2.95$-fold higher in the SKPSH10 group than in the PSH, SPSH, and KPSH10 groups, respectively. Therefore, the hydrogel loaded with SDF-1 and KGN successfully promoted chondrogenic differentiation.

\section{Macroscopic evaluation of regenerated cartilage}

The macromorphology of regenerated cartilage was evaluated by optical microscopy after the operation (Fig. 9A). At 4 and 12 weeks, none of the groups showed any obvious signs of infection. After 4 weeks, all materials had been degraded. The MF and PSH groups showed obvious cavities, small amounts of new tissue and an obvious boundary with the surrounding normal tissue. However, there was a great deal of new tissue formation in the defect area with surface roughness and obvious boundaries in the SPSH and KPSH10 groups. However, the defect area was covered by hyaline tissue, and the surface with slight cracks did not show an obvious boundary with surrounding native tissue. At 12 weeks after the operation, the regenerated tissue filled the defect area with obviously cracked surfaces in the MF, PSH, SPSH, and KPSH10 groups. The new tissue completely filled defects with a smooth surface and was tightly combined with the surrounding tissue in the SKPSH10 group. The macroscopic score results in Fig. 9B clearly show a better regeneration effect in the SKPSH10 group than in the other groups.

\section{Recruitment of rBMSCs in vivo}

The recruitment of rBMSCs by hydrogel was evaluated by staining for CD44 and CD90 at 7 days after implantation, as 

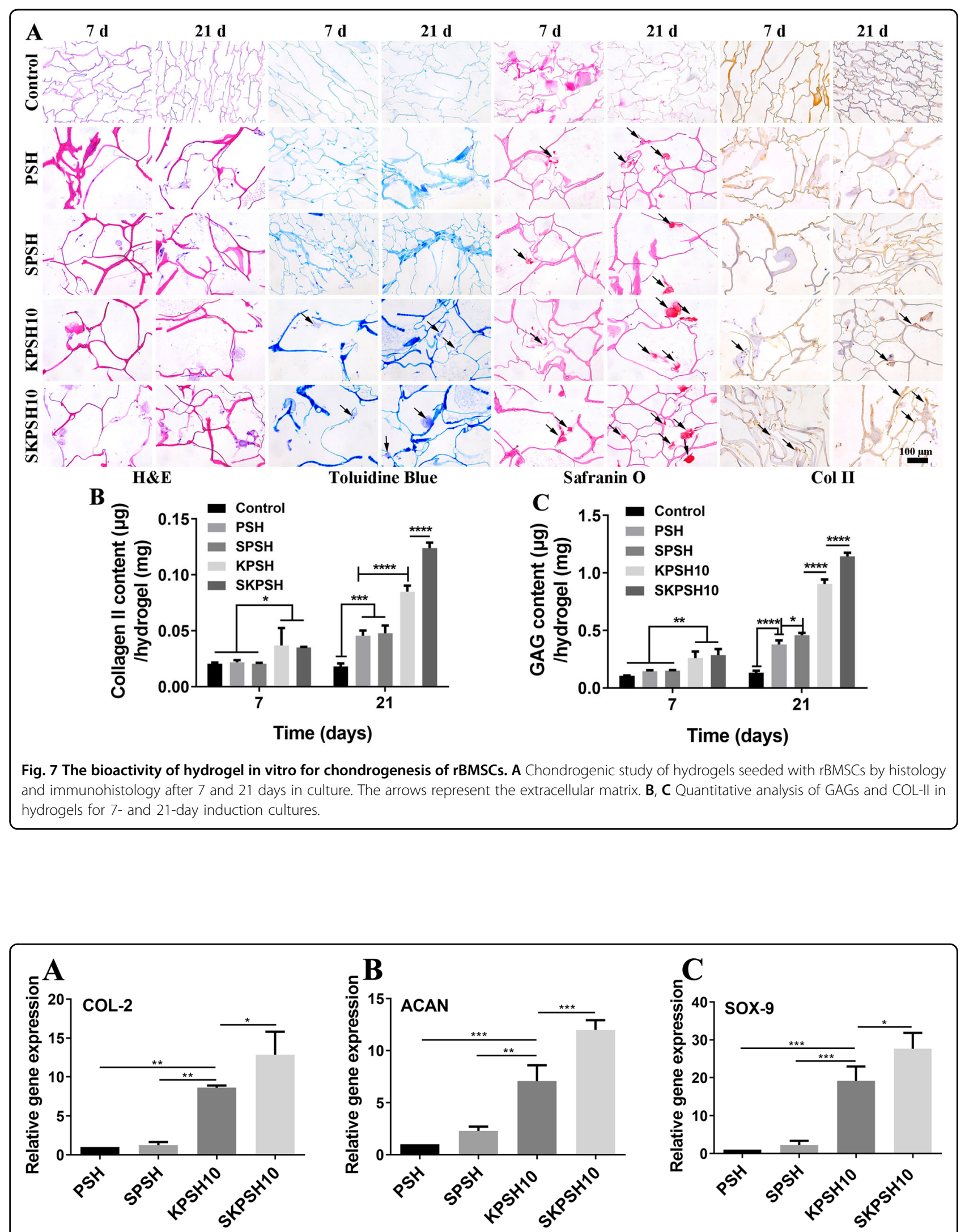

Fig. 8 Cartilage-related gene expression in hydrogel-seeded rBMSCs after $\mathbf{2 1}$ days of culture. A COL-II mRNA levels. B ACAN mRNA levels. C SOX-9 mRNA levels. 


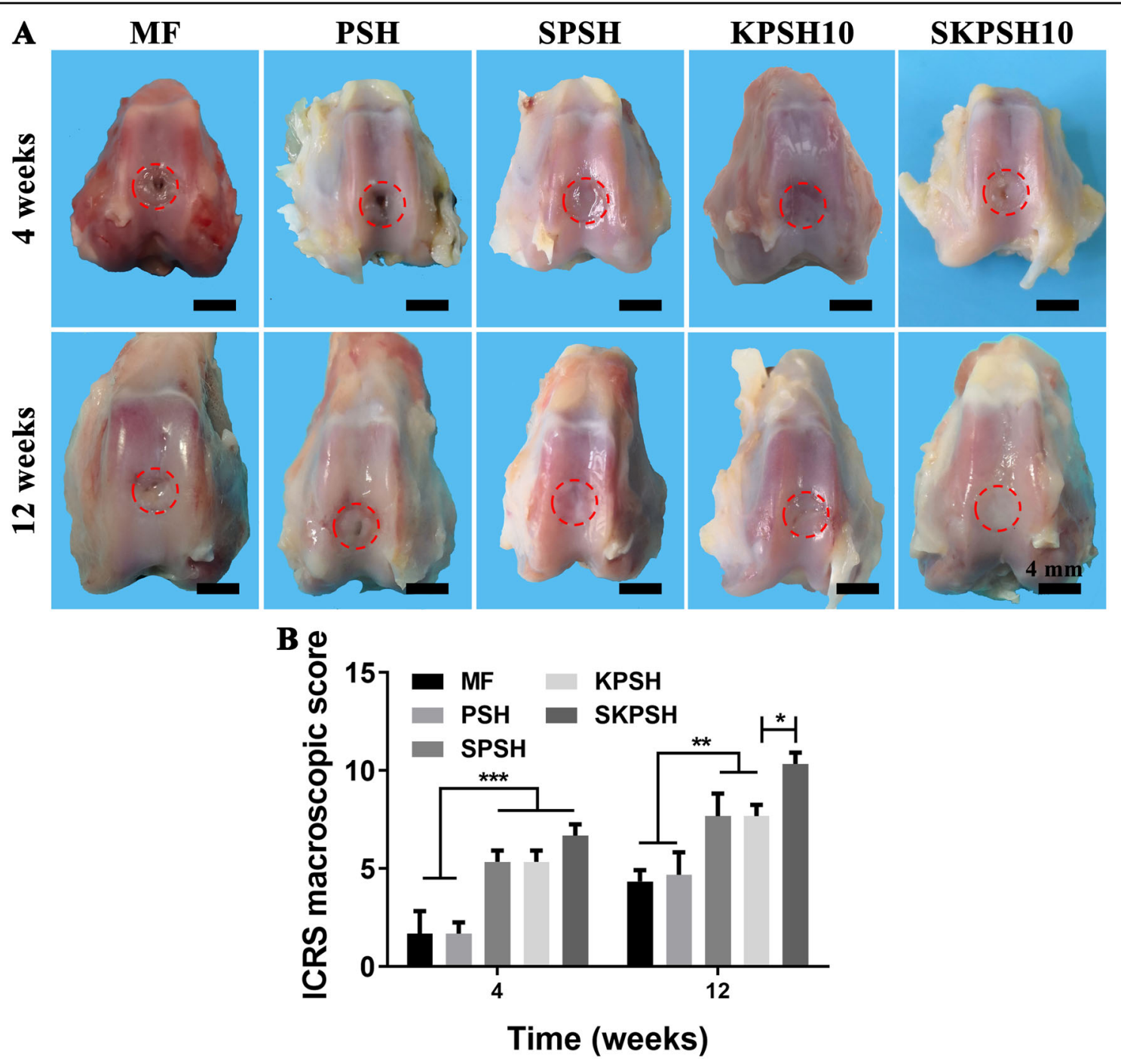

Fig. 9 Gross observation and score of new cartilage at $\mathbf{4}$ and 12 weeks after operation. A Gross observation. B Macroscopic score.

shown in Fig. 10. The results shown in Fig. 10A indicated that CD44 (green) and CD90 (red) were positively expressed in the defect area. The fluorescence intensities for CD44 and CD90 on rBMSCs in the SDF-1 groups (SPSH and SKPSH10 groups) were significantly more obvious than those in other groups (i.e., the MF, PSH, and KPSH10 groups). The quantitative results in Fig. $10 \mathrm{~B}$ and $\mathrm{C}$ clearly show that the number of CD44- and CD90-positive cells in the SPSH group and the SKPSH10 group was greater than that in the other groups. However, there was no significant difference between the SPSH group and the SKPSH10 group. These results further indicated that SDF-1 effectively promoted the recruitment of MSCs in vivo.

\section{Restoration of cartilage defects in vivo}

The results of H\&E staining are shown in Fig. 11. At 4 weeks after the operation, hydrogels in all groups were completely degraded in the defect area. In MF, PSH, $\mathrm{SPSH}$, and KPSH10, there were still defects in the subchondral bone, and fibrous tissues filled the defect area. However, the SKPSH10 group formed continuous subchondral bone, and the defect in subchondral bone caused by microfracture was reconstructed. In the MF and PSH groups, the regenerated cartilage tissues were less abundant and were discontinuous. The new tissues filling the defect areas in the SPSH and KPSH10 groups were more continuous but were still not smooth. The SKPSH10 group showed continuous new tissues in the cartilage defect area with a regular cell arrangement. Even so, the new tissues and natural tissue structure were still significantly different and had clear boundaries in all groups. At 12 weeks, the subchondral bones of all groups were basically reconstructed, but the reconstruction of subchondral bones in the KPSH10 group and SKPSH10 

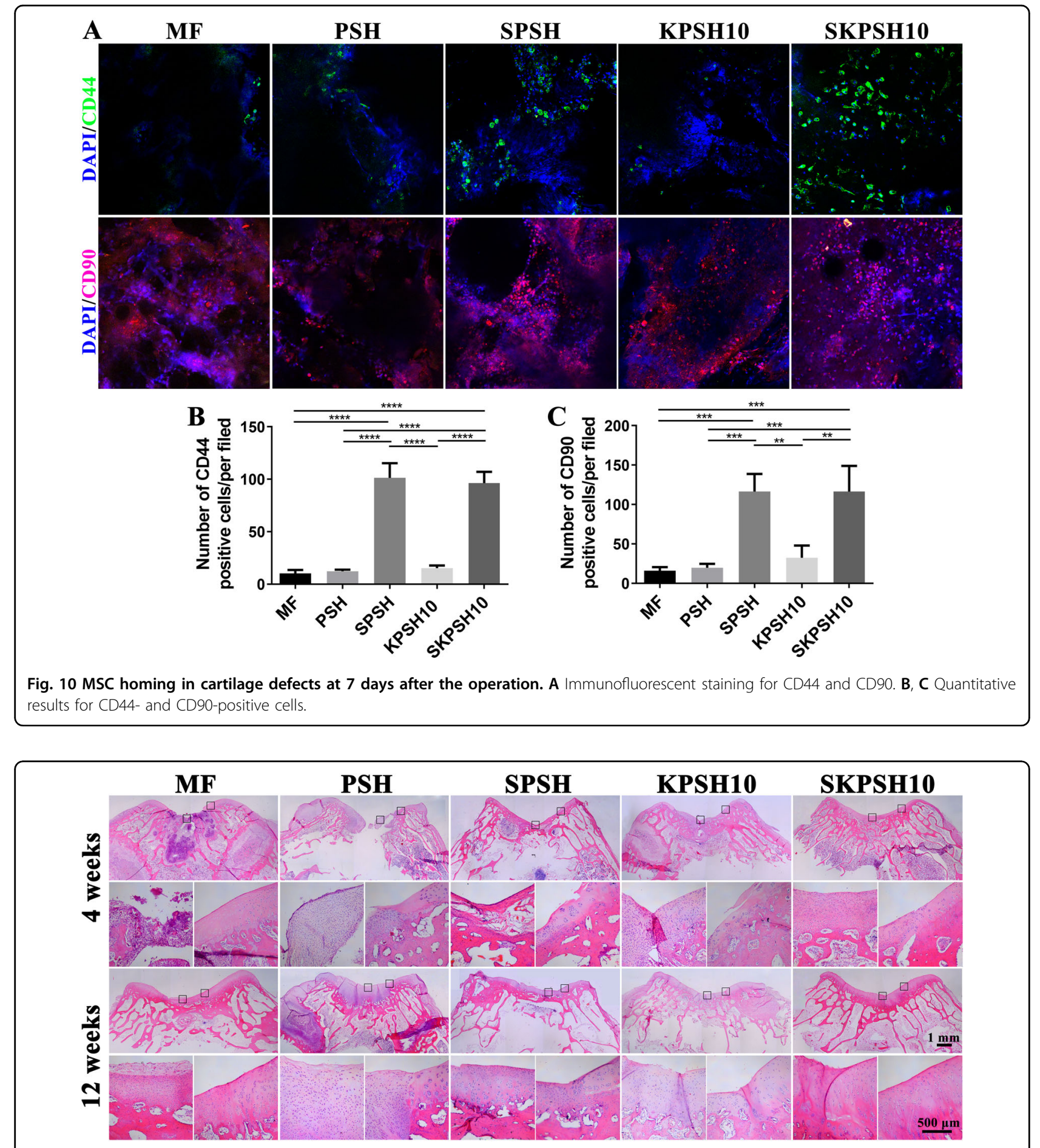

Fig. $11 \mathrm{H} \& \mathrm{E}$ staining for regenerated cartilage in defects at 4 and 12 weeks postoperatively. Rectangular boxes on the left and right indicate new tissue and defect edges, respectively.

group showed more formation of trabeculae, which was more similar to the natural subchondral bone. Furthermore, the cartilage defect areas of every group were further filled with new tissues, especially in the SPSH, KPSH10, and SKPSH10 groups, which showed obvious regeneration of cartilage tissues. More continuous cartilage structure and mature chondrocyte morphology were observed in the SKPSH10 group than in the other groups. Some chondrocytes were inlaid in the cartilage lacuna, indicating a good regeneration effect. 


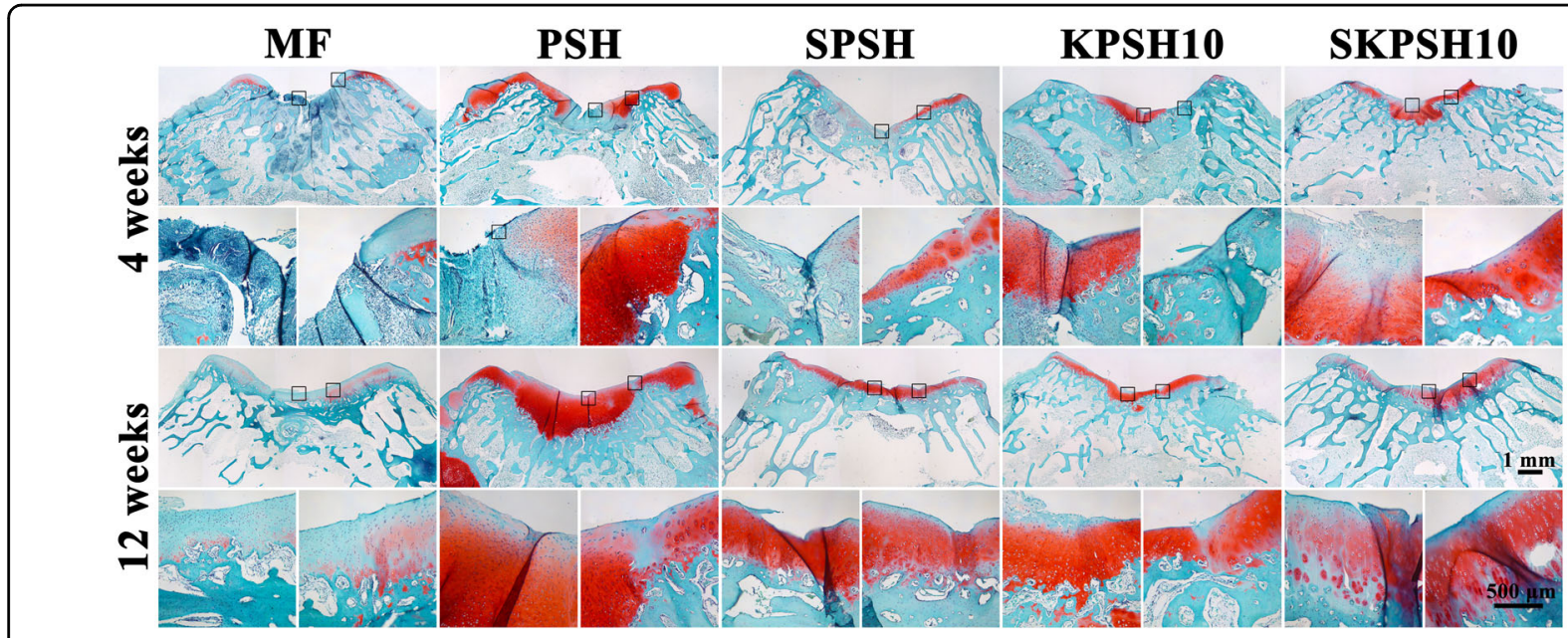

Fig. 12 Safranin-O/fast green staining for regenerated cartilage in the defects at $\mathbf{4}$ and 12 weeks postoperatively. Rectangular boxes on the left and right indicate new tissues and defect edges, respectively.

Staining with safranin-O/fast green was performed to further visualize the regenerated cartilage tissues in the various groups, as shown in Fig. 12. Safranin-O/fast green stained the normal cartilage and subchondral bone light red and light green, respectively. At 4 weeks, no red staining was observed in the defect area in the MF, PSH, or SPSH groups. Fibrous tissues were formed, while the newly formed tissue surfaces in the KPSH10 and SKPSH10 groups showed partial red staining, which indicated the generation of cartilage extracellular matrix (CECM) rich in glycosaminoglycan (GAG), but the new CECM was more continuous in the SKPSH group. At 12 weeks, defect repair was significantly improved in all groups compared with that at 4 weeks, but the regenerated tissue in the MF group was filled with fibrous tissues without GAG formation, indicating that the animals had insufficient self-healing capacity to repair the cartilage in the defect sites. In the PSH, SPSH, KPSH10, and SKPSH10 groups, there were large amounts of CECM deposition containing GAG. In particular, the cartilage showed a continuous structure with a smooth surface in the SPSH, KPSH10, and SKPSH10 groups, similar to the surrounding natural tissues. Moreover, chondrocytes in the SKPSH10 group were closer in morphology, arrangement and distribution to the natural tissue. The results showed that the SKPSH10 group had advantages in the promotion of cartilage defect repair.

The results of immunohistochemical analysis at 4 and 12 weeks showed that positivity for COL-II expression was more continuous in the SKPSH10 group than in the other groups (Fig. 13), indicating an advantage with regard to the promotion of ECM generation and cartilage regeneration in this group. The regenerated cartilage tissue in the SKPSH10 group with uniform features showed no boundary with the surrounding native cartilage tissue, as well as a smooth surface with abundant COL-II. The chondrocytes in the regenerated cartilage showed a typical lacunar structure.

With respect to the histological scores for cartilage evaluation in Fig. 14, the SPSH, KPSH, and SKPSH groups at 4 weeks and at 12 weeks had dramatically higher scores than the MF group at both time points. In particular, the SKPSH group had a higher score than the other groups, which showed that the spatiotemporal release of SDF-1 and KGN could shorten the process of cartilage regeneration. There was no significant difference among the SPSH, KPSH, and SKPSH groups at 12 weeks. The results further and intuitively indicated that sequentially releasing SDF-1 and KGN in vivo had good bioactivity and therapeutic effects. Thus, the hydrogel with the sequential release of SDF-1 and KGN could enhance cartilage regeneration.

\section{Discussion}

There has been a great deal of research interest in cellfree strategies involving the combination of biomaterials with endogenous stem cells for the repair of cartilage defects in the field of $\mathrm{TE}^{20,42}$. BMSCs and other endogenous cells play important roles in cartilage regeneration, but the limited migration of endogenous stem cells and their short-term retention in local tissues limit their roles in cartilage repair ${ }^{36,39}$. An ideal material for cartilage regeneration would recruit sufficient stem cells from subchondral bone and enhance their retention capability at the sites of cartilage defects ${ }^{43}$. Previous studies showed that SDF-1 could promote the recruitment and migration of $\mathrm{MSCs}^{44,45}$. In addition, it is important to maintain the microenvironment of chondrogenic differentiation for defect repair. As a small-molecule compound, KGN was shown to promote the chondrogenic differentiation of stem cells ${ }^{46}$. SDF-1 has low bioavailability due to its rapid 


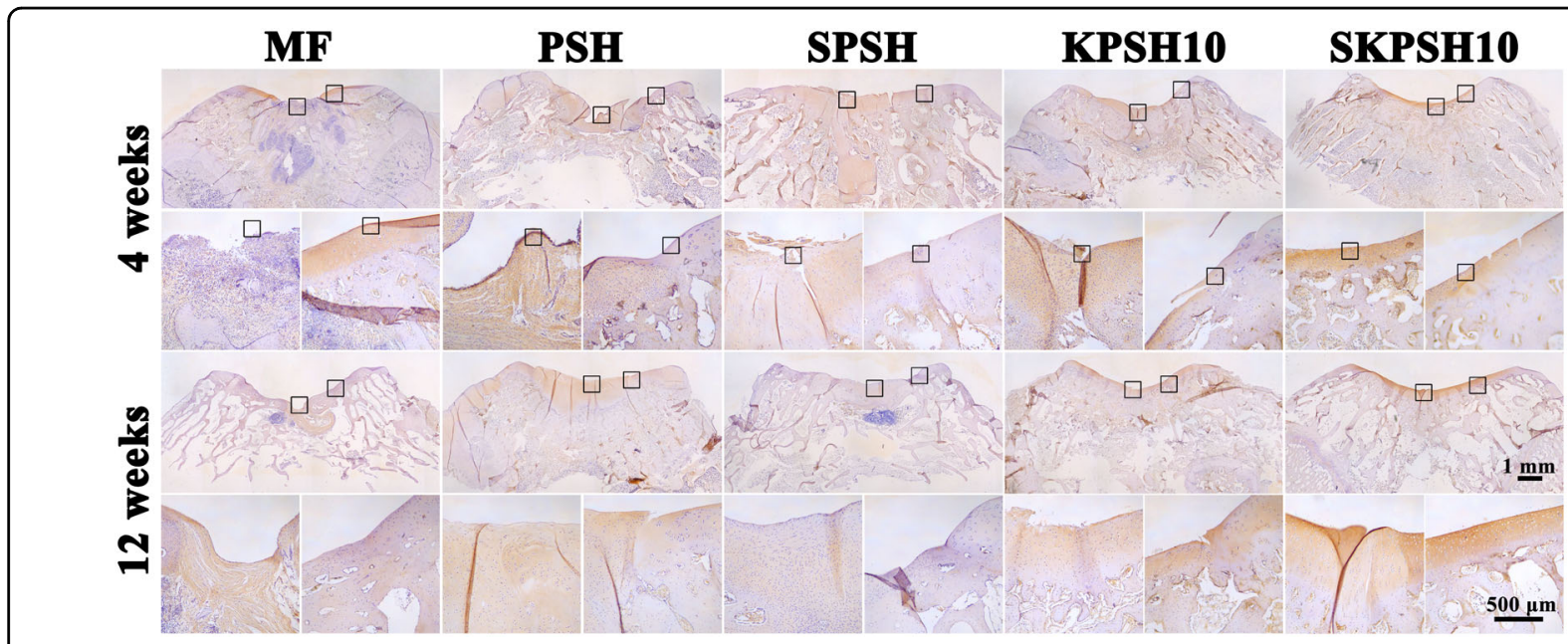

Fig. 13 Immunohistochemical analysis of regenerated cartilage in the defects at 4 and 12 weeks postoperatively. Rectangular boxes on the left and right indicate new tissues and defect edges, respectively.
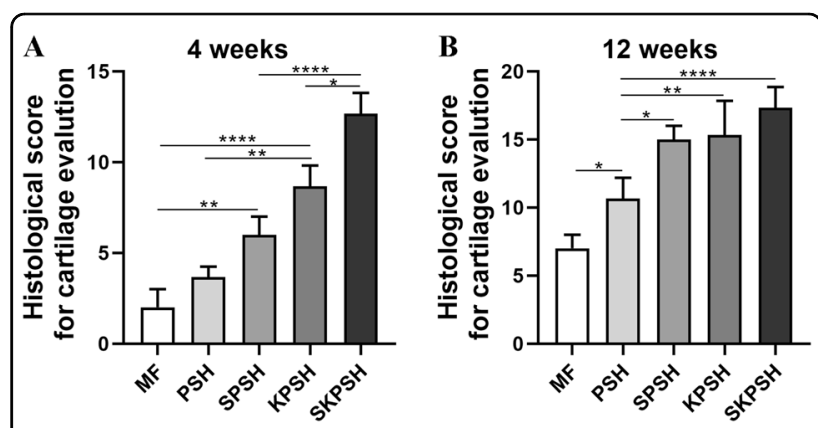

Fig. 14 Histological score for regenerated cartilage in the defects at 4 and 12 weeks postoperatively. A Histological score at 4 weeks. B Histological score at 12 weeks.

diffusion and short half-life ${ }^{47}$, and KGN is insoluble and has potential side effects ${ }^{48}$. To resolve these issues, a number of studies have investigated drug delivery modes involving the direct loading of drugs into biomaterials or encapsulation in microspheres, which could improve the bioavailability of drugs and reduce their side effects in vivo ${ }^{49,50}$. It is important to develop drug delivery systems to effectively maintain bioactive substances at relatively constant drug release rates to enhance therapeutic effects. Previous studies have shown that the sequential release of drugs is more conducive to cartilage repair ${ }^{51}$. Therefore, we developed an injectable hydrogel capable of the sequential release of SDF-1 and KGN to form bifunctional materials (Figs. 2-4).

CS has active amino groups, which are suitable for chemical modification ${ }^{52}$. PC was formed by grafting PA to the CS side chain by chemical modification (Fig. 2). The phenolic hydroxyl groups in PC and the tyrosine contained in SF were crosslinked to form a hydrogel catalyzed by HRP and hydrogen peroxide ${ }^{53}$. Therefore, PC and SF could form a variety of cross-linkages to promote hydrogel formation, including PC-PC, PC-SF, and SF-SF bonds. In clinical cases, the shape and depth of cartilage defects are usually diverse 9 . PC/SF composite hydrogels are injectable and can be readily formed into different shapes (Fig. 4A1-D1, Fig. S2 and Fig. S3). However, with SF contents $>90 \%$, hydrogels could not be formed, as the paucity of phenolic hydroxyl groups in the mixed solution inhibited the occurrence of the crosslinking reaction (Fig. S2). As a TE material, CS has been widely used in the preparation of hydrogels, but the mechanical properties of CS alone are poor and do not meet clinical requirements ${ }^{18}$. SF has good mechanical properties and can improve the mechanical strength of hydrogels ${ }^{54}$. The results indicated that the hydrogel with a mass ratio of 1:5 for PC and SF has good mechanical properties (Fig. S6). Hydrogels can be used as drug and cell carriers to facilitate uniform distribution and can be adapted to fit cartilage defects of various sizes and shapes. Microscopic observations indicated that the hydrogels had a uniform reticular porous structure, which could provide a good 3D microenvironment for cell survival and function (Fig. 4A2-D2 and Fig. A3-D3).

The sequential release models were formed by the rapid release of SDF-1 and the sustained release of KGN (Fig. 4F). The different release behaviors of SDF-1 and KGN were related to the encapsulation mode; the tighter encapsulation of KGN by microspheres resulted in later release than that of SDF-1, thus allowing sequential drug release. In addition, KGN is a fat-soluble drug, while SDF1 is hydrophilic; thus, the dissolution characteristics represent another reason for the sequential release of KGN and SDF-1. Therefore, we hypothesized that the sequential release of these two bioactive molecules from hydrogels is beneficial for spatiotemporally regulating the 
biological behavior of MSCs to promote cartilage regeneration because the rapid release of SDF-1 promotes MSC homing to the defect areas in the early stage, whereas the sustained release of KGN maintains an effective concentration of KGN to promote the transformation of MSCs into chondrocytes. To test this hypothesis and its potential application in cartilage defects, the in vitro cell viability, cell migration, and chondrogenic differentiation of rBMSCs, as well as in vivo cartilage formation, were assessed.

The selection and preparation of materials can directly affect the cytocompatibility of hydrogels. The hydrogel used in the present study had good cytocompatibility (Fig. 5, Fig. S10 and Fig. S11). The cells seeded in the hydrogel showed good viability and adhesion, and the addition of microspheres did not have any negative effects. SF, CS, and PLGA have good cytocompatibility, as does the method of crosslinking by enzyme catalysis, which could further ensure safety. Moreover, the structures of the hydrogels were similar to that of the ECM and thus could provide a suitable 3D microenvironment for cell survival, facilitate the transport of cell metabolites and nutrients, and promote cell adhesion, proliferation, and differentiation ${ }^{55}$.

In vitro rBMSC migration assays (Fig. 6) and induction differentiation assays (Figs. 7 and 8) were performed to evaluate the functionality of SDF-1 and KGN released from the hydrogel. Ji et al. reported that the loading concentration of SDF- 1 on the material at $50-400 \mathrm{ng} / \mathrm{mL}$ could have a significant chemotactic effect on MSCs, and the effect was not dose dependent ${ }^{56}$. Therefore, $200 \mathrm{ng} / \mathrm{mL}$ SDF-1 was used in the hydrogel in the present study. In this study, the SPSH and SKPSH groups showed promotion of rBMSC migration, indicating that the hydrogel-released SDF-1 had good activity. In addition, the KPSH10 and SKPSH10 groups loaded with KGN showed promotion of chondrocyte matrix formation and the expression of related genes after 7 and 21 days of induction culture in vitro, but the effect was most significant in the SKPSH10 group. The results indicated that the effect of KGN on chondrocyte differentiation was enhanced by SDF-1. It has been reported that SDF-1 inhibits the production of collagen I (COL-I) and matrix metalloproteinase-13 (MMP-13) ${ }^{24}$. SDF-1 inhibits COL-I synthesis, which is beneficial for the protection of chondrocytes from ossification ${ }^{57}$. Therefore, SDF-1 enhances chondrogenic differentiation of rBMSCs.

The results of in vivo experiments indicated the most significant effects on MSC recruitment and cartilage repair in the SKPSH10 group (Figs. 9-14). The results reported by Yiting Wen et al. have shown that stem cells or progenitor cells in the neighborhood of the cartilage defect area may respond to SDF-1 and migrate into the injured portion to perform their function ${ }^{51}$. Moreover, CD44 and CD90 were used as stem cell markers to validate their recruitment in vitro ${ }^{58}$ and vivo ${ }^{59}$. Therefore, the SDF-1 released by hydrogel could first fully exhibit MSC homing ability and cause cells to migrate to the defect areas. MSCs then underwent chondrogenic differentiation upon stimulation with $\mathrm{KGN}$, thus promoting cartilage defect repair. The migration and chondrogenic differentiation of endogenic cells, such as BMSCs, play key roles in cartilage regeneration, but poor migration and short local retention restrict their use in cartilage repairing $^{51,60}$. Thus, the sequential release of SDF-1 and KGN could promote the migration of endogenous stem cells to the defect area and ensure adequate stem cells for further repair by the chondrogenic differentiation of MSCs. This system showed a number of unique advantages in promoting cartilage regeneration.

\section{Conclusion}

In this study, a cell-free hydrogel system was designed to recruit endogenous stem cells and induce their differentiation into chondrocytes. The system used PC and SF in combination with SDF-1 and PLGA microspheres loaded with KGN to form an injectable hydrogel, which showed sequential and sustained drug release. The hydrogel prepared here has a reticulated porous structure and good injection performance, which are conducive to filling irregular cartilage defects. The hydrogel released SDF-1 quickly and KGN slowly, showing a good sequential release profile. The hydrogel system had good biocompatibility, promoted the recruitment and differentiation of stem cells to chondrocytes and enhanced the regeneration of articular cartilage in situ. Based on these results, we conclude that injectable hydrogels with sequential release of SDF-1 and KGN have good therapeutic potential for promoting cartilage regeneration and may be useful in practical applications in the future.

\section{Acknowledgements \\ This work was dedicated to the 100th anniversary of Chemistry at Nankai University.}

\section{Author details}

${ }^{1}$ State Key Laboratory of Medicinal Chemical Biology; The Key Laboratory of Bioactive Materials, The College of Life Science, Nankai University, 300071 Tianjin, China. ${ }^{2}$ Department of Spine Surgery, Tianjin Hospital, Tianjin University, 300211 Tianjin, China. ${ }^{3}$ Tianjin Stomatological Hospital, School of Medicine, Nankai University, 300041 Tianjin, China

\section{Author contributions}

Y.D., Q.Y., and S.W. designed the research; Q.Y. and S.W. supervised the project; Y.D., Y.L., and Y.C. performed the experiments; Y.D. and S.W. prepared the manuscript; L.Z., X.S., Z.Z., Y.W., and C.Q. contributed to the discussion, language improvements, and proofreading.

\section{Funding information}

This work was supported by the National Natural Science Foundation of China (31870966, 82072435, 81800931, 81901905, 81901062), the National Key Research Development Program of China (2017YFC1103504,

2020YFA0803701), the Tianjin Science Foundation (20YFZCSY01020), and the Reach Foundation of the Tianjin Health Commission (KJ20052). 


\section{Competing interests}

The authors declare no competing interests.

\section{Publisher's note}

Springer Nature remains neutral with regard to jurisdictional claims in published maps and institutional affiliations.

Supplementary information The online version contains supplementary material available at https://doi.org/10.1038/s41427-021-00339-3.

Received: 6 July 2021 Revised: 29 September 2021 Accepted: 6 October 2021.

Published online: 19 November 2021

\section{References}

1. Rogan, H., llagan, F., Tong, X., Chu, C. R. \& Yang, F. Microribbon-hydrogel composite scaffold accelerates cartilage regeneration in vivo with enhanced mechanical properties using mixed stem cells and chondrocytes. Biomaterials 228, 119579 (2020)

2. $\mathrm{Xu}$, L. et al. Metformin hydrochloride encapsulation by alginate strontium hydrogel for cartilage regeneration by reliving cellular senescence. Biomacromolecules 22, 671-680 (2021).

3. $\mathrm{Hu}, \mathrm{H}$. et al. miR-23a-3p-abundant small extracellular vesicles released from GelMA/nanoclay hydrogel for cartilage regeneration. J. Extracell. Vesicles 9, 1778883 (2020).

4. Dong, $Y$. et al. Regional and sustained dual-release of growth factors from biomimetic tri-layered scaffolds for the repair of large-scale osteochondral defects. Appl. Mater. Today 19, 100548 (2020).

5. Zhao, Y. et al. Role of the calcified cartilage layer of an integrated trilayered silk fibroin scaffold used to regenerate osteochondral defects in rabbit knees. ACS Biomater. Sci. Eng. 6, 1208-1216 (2020).

6. Liu, J. et al. Cell-free scaffolds functionalized with bionic cartilage acellular matrix microspheres to enhance the microfracture treatment of articular cartilage defects. J. Mater. Chem. B 9, 1686-1697 (2021).

7. Howell, M., Liao, Q. \& Gee, C. W. Surgical management of osteochondral defects of the knee: an educational review. Curr. Rev. Musculoskelet Med. 14, 60-66 (2021).

8. Baranowski, M. \& Czubak, J. New perspectives in joint cartilage treatment with special emphasis on new types of hydrogels: a review. Ortop. Traumatol. Rehabil. 21, 237-251 (2019).

9. Kwon, H. et al. Surgical and tissue engineering strategies for articular cartilage and meniscus repair. Nat. Rev. Rheumatol. 15, 550-570 (2019).

10. Dang, Q. et al. Fabrication and evaluation of thermosensitive chitosan/collagen/a, $\beta$-glycerophosphate hydrogels for tissue regeneration. Carbohydr. Polym. 167, 145-157 (2017).

11. Li, Z., Wang, F., Roy, S., Sen, C. K. \& Guan, J. Injectable, highly flexible, and thermosensitive hydrogels capable of delivering superoxide dismutase. Biomacromolecules 10, 3306-3316 (2009).

12. Bahareh et al. Novel thermosensitive hydrogel composites based on poly (d,l-lactide-co-glycolide) nanoparticles embedded in poly(n-isopropyl acrylamide) with sustained drug-release behavior. J. Appl. Polym. Sci. 131, 40625 (2014)

13. Byeongmoon et al. Thermosensitive sol-gel reversible hydrogel. Adv. Drug Deliv. Rev. 64, 154-162 (2012).

14. Soni, S. S. \& Rodell, C. B. Polymeric materials for immune engineering: molecular interaction to biomaterial design. Acta Biomater. 12, 2404 (2021).

15. Nandi, S. K., Kundu, B. \& Basu, D. Protein growth factors loaded highly porous chitosan scaffold: a comparison of bone healing properties. Mater. Sci. Eng. C. Mater. Biol. Appl. 33, 1267-1275 (2013).

16. LogithKumar, R. et al. A review of chitosan and its derivatives in bone tissue engineering. Carbohydr. Polym. 151, 172-188 (2016).

17. Costa-Pinto, A. R., Reis, R. L. \& Neves, N. M. Scaffolds based bone tissue engineering: the role of chitosan. Tissue Eng. Part. B Rev. 17, 331-347 (2011).

18. Huang, $H$. et al. Directing chondrogenic differentiation of mesenchymal stem cells with a solid-supported chitosan thermogel for cartilage tissue engineering. Biomed. Mater. 9, 035008 (2014).
19. Holland, C., Numata, K, Rnjak-Kovacina, J. \& Seib, F. P. The biomedical use of silk: past, present, future. Adv. Healthc. Mater. 8, e1800465 (2019).

20. Sun, $X$. et al. In Situ Articular cartilage regeneration through endogenous reparative cell homing using a functional bone marrow-specific scaffolding system. ACS Appl. Mater. Inter. 10, 38715-38728 (2018).

21. Lee, C. H. et al. Regeneration of the articular surface of the rabbit synovial joint by cell homing: a proof of concept study. Lancet 376, 440-448 (2010).

22. Zhang, W. et al. An all-silk-derived functional nanosphere matrix for sequential biomolecule delivery and in situ osteochondral regeneration. Bioact. Mater. 5, 832-843 (2020).

23. Lee, D. et al. Sequential dual-drug delivery of BMP-2 and alendronate from hydroxyapatite-collagen scaffolds for enhanced bone regeneration. Sci. Rep. 11,746 (2021).

24. Chen, P. et al. Radially oriented collagen scaffold with SDF-1 promotes osteochondral repair by facilitating cell homing. Biomaterials 39, 114-123 (2015).

25. Theiss, H. D. et al. Dual stem cell therapy after myocardial infarction acts specifically by enhanced homing via the SDF-1/CXCR4 axis. Stem Cell Res. 7, 244-255 (2011).

26. Ono, Y., Ishizuka, S., Knudson, C. B. \& Knudson, W. Chondroprotective effect of kartogenin on CD44-mediated functions in articular cartilage and chondrocytes. Cartilage 5, 172-180 (2014).

27. Johnson, K. et al. A stem cell-based approach to cartilage repair. Science $\mathbf{3 3 6}$ 717-721 (2012).

28. $\mathrm{Xu}$, J. et al. Injectable stem cell-laden supramolecular hydrogels enhance in situ osteochondral regeneration via the sustained co-delivery of hydrophilic and hydrophobic chondrogenic molecules. Biomaterials 210, 51-61 (2019).

29. Sinha, V. R. \& Trehan, A. Biodegradable microspheres for protein delivery. J. Control. Release 90, 261-280 (2003).

30. Xu, X. et al. Exosome-mediated delivery of kartogenin for chondrogenesis of synovial fluid-derived mesenchymal stem cells and cartilage regeneration. Biomaterials 269, 120539 (2020).

31. Hu, Q. et al. Polyethylene glycol modified PAMAM dendrimer delivery of kartogenin to induce chondrogenic differentiation of mesenchymal stem cells. Nanomedicine 13, 2189-2198 (2017).

32. Sulaiman, S. B., Idrus, R. B. H. \& Hwei, N. M. Gelatin microsphere for cartilage tissue engineering: current and future strategies. Polymers (Basel) 12, 2404 (2020).

33. Sun, X., Wang, J., Wang, Y. \& Zhang, Q. Collagen-based porous scaffolds containing PLGA microspheres for controlled kartogenin release in cartilage tissue engineering. Artif. Cell. Nanomed. B. 46, 1957-1966 (2018).

34. Matsiko, A., Levingstone, T. J. \& O'Brien, F. J. Advanced strategies for articular cartilage defect repair. Materials (Basel) 6, 637-668 (2013).

35. Teng, B. et al. A chondrogenesis induction system based on a functionalized hyaluronic acid hydrogel sequentially promoting hMSC proliferation, condensation, differentiation, and matrix deposition. Acta Biomater. 122, 145-159 (2021).

36. Barry, F. \& Murphy, M. Mesenchymal stem cells in joint disease and repair. Nat. Rev. Rheumatol. 9, 584-594 (2013).

37. Sarkaria, S. M., Decker, M. \& Ding, L. Bone marrow micro-environment in normal and deranged hematopoiesis: opportunities for regenerative medicine and therapies. Bioessays 40, 10 (2018).

38. Pourrajab, F., Forouzannia, S. K. \& Tabatabaee, S. A. Molecular characteristics of bone marrow mesenchymal stem cells, source of regenerative medicine. Int. J. Cardiol. 163, 125-131 (2013).

39. Huang, $\mathrm{H}$. et al. A functional biphasic biomaterial homing mesenchymal stem cells for in vivo cartilage regeneration. Biomaterials $\mathbf{3 5}$, 9608-9619 (2014)

40. Steadman, J. R. et al. Outcomes of microfracture for traumatic chondral defects of the knee: average 11-year follow-up. Arthroscopy 19, 477-484 (2003).

41. Liu, T. et al. Kartogenin mediates cartilage regeneration by stimulating the IL6/Stat3-dependent proliferation of cartilage stem/progenitor cells. Biochem. Biophys. Res. Commun. 532, 385-392 (2020).

42. Shi, W. et al. Structurally and functionally optimized silk-fibroin-gelatin scaffold using 3D printing to repair cartilage injury in vitro and in vivo. Adv. Mater. 29, 1701089 (2017).

43. Reichert, J. C. et al. A tissue engineering solution for segmental defect regeneration in load-bearing long bones. Sci. Transl. Med. 4, 141 ra93 (2012).

44. Gonçalves, R. M., Antunes, J. C. \& Barbosa, M. A. Mesenchymal stem cell recruitment by stromal derived factor-1-delivery systems based on chitosan/ poly(Y-glutamic acid) polyelectrolyte complexes. Eur. Cell. Mater. 23, 249-260 (2012). 
45. Chen, $Y$. et al. Attenuation of subchondral bone abnormal changes in osteoarthritis by inhibition of SDF-1 signaling. Osteoarthr. Cartil. 25, 986-994 (2017).

46. Shi, D. et al. Photo-cross-linked scaffold with kartogenin-encapsulated nanoparticles for cartilage regeneration. ACS Nano 10, 1292-1299 (2016).

47. Mi, L., Liu, H., Gao, Y., Miao, H. \& Ruan, J. Injectable nanoparticles/hydrogels composite as sustained release system with stromal cell-derived factor-1a for calvarial bone regeneration. Int. J. Biol. Macromol. 101, 341-347 (2017).

48. Zare, P. et al. Alginate sulfate-based hydrogel/nanofiber composite scaffold with controlled Kartogenin delivery for tissue engineering. Carbohydr. Polym. 266, 118123 (2021).

49. Lam, J., Lu, S., Kasper, F. K. \& Mikos, A. G. Strategies for controlled delivery of biologics for cartilage repair. Adv. Drug. Deliv. Rev. 84, 123-134 (2015).

50. Mottaghitalab, F., Farokhi, M., Shokrgozar, M. A., Atyabi, F. \& Hosseinkhani, H. Silk fibroin nanoparticle as a novel drug delivery system. J. Control. Release $\mathbf{2 0 6}$ 161-176 (2015).

51. Wen, Y. T., Dai, N. T. \& Hsu, S. H. Biodegradable water-based polyurethane scaffolds with a sequential release function for cell-free cartilage tissue engineering. Acta Biomater. 88, 301-313 (2019).

52. Sultankulov, B., Berillo, D., Sultankulova, K., Tokay, T. \& Saparov, A. Progress in the development of chitosan-based biomaterials for tissue engineering and regenerative medicine. Biomolecules 9, 470 (2019).
53. Su, D. et al. Enhancing mechanical properties of silk fibroin hydrogel through restricting the growth of $\beta$-sheet domains. ACS Appl. Mater. Inter. 9, 17489-17498 (2017).

54. Lu, Q., Zhang, X., Hu, X. \& Kaplan, D. L. Green process to prepare silk fibroin/ gelatin biomaterial scaffolds. Macromol. Biosci. 10, 289-298 (2010).

55. Dong, $\mathrm{Y}$. et al. Poly(N-isopropyl-acrylamide)/poly(Y-glutamic acid) thermosensitive hydrogels loaded with superoxide dismutase for wound dressing application. Int. J. Nanomed. 15, 1939-1950 (2020).

56. Ji, W. et al. Incorporation of stromal cell-derived factor-1a in PCL/gelatin electrospun membranes for guided bone regeneration. Biomaterials 34 735-745 (2013).

57. Han, M., Yang, X., Lee, J., Allan, C. H. \& Muneoka, K. Development and regeneration of the neonatal digit tip in mice. Dev. Biol. 315, 125-135 (2008).

58. Zhang, Q. et al. Effects of the fibrous topography-mediated macrophage phenotype transition on the recruitment of mesenchymal stem cells: an in vivo study. Biomaterials 149, 77-87 (2017).

59. Zhang, H., Yu, S., Zhao, X., Mao, Z. \& Gao, C. Stromal cell-derived factor-1aencapsulated albumin/heparin nanoparticles for induced stem cell migration and intervertebral disc regeneration in vivo. Acta Biomater. 72, 217-227 (2018).

60. Chen, Y. et al. Sustained release SDF-1a/TGF- $\beta 1$-loaded silk fibroin-porous gelatin scaffold promotes cartilage repair. ACS Appl. Mater. Inter. 11, 14608-14618 (2019). 